ORIGINAL ARTICLE

\title{
Detection of non-accidental injuries presenting at emergency departments
}

\author{
A McKinney, G Lane, F Hickey
}

See end of article for

authors' affiliations

Emerg Med J 2004;21:562-564. doi: 10.1136/emj.2003.008607

Correspondence to:

Dr A McKinney,

Department of Mental

Health, Queens University

Belfast, Whitla Medical

Building, 97 Lisburn Road,

Belfast BT9 7BL, UK;

adelemckinney@

hotmail.com

Accepted for publication 13 October 2003

\begin{abstract}
Objectives: To investigate whether cases of possible non-accidental injury as identified using five risk indicators give rise to any subjective concerns of child abuse.

Methods: Questionnaires were completed by the triage nurse and attending doctor for every child attending the general hospitals of the North Western Health Board, with an injury, during a six month period. The questionnaires included an assessment of subjective concerns about the injury occurrence and five risk indicators of child abuse.

Results: Children presenting with an injury who had two or more positive indicators failed to raise subjective concerns in the attending emergency department staff.

Conclusions: The introduction of a policy of identifying positive indicators from the five risk indicators of child abuse needs additional computer support within emergency departments.
\end{abstract}

n children, injury mortality is greater than childhood mortality from all other causes combined. ${ }^{1}$ In the mid-

1940s Dr John Caffey identified unexplained fractures in children presenting to emergency departments with other injuries. This finding was primarily attributable to advances in radiological technology and led to the conceptualisation of "the battered child syndrome". ${ }^{2}$ Emergency physicians are often placed in a critical role in the lives of individuals and have daily exposure to high risk populations. This gives emergency physicians a unique opportunity to take an active role in injury control and prevention. Recently research has investigated the possibility of identifying non-accidental injury (NAI) in the emergency department. Benger and $\mathrm{McCabe}^{3}$ introduced a change in policy in the form of a routine reminder mechanism into the accident and emergency notes, coupled with an improved programme of NAI education and awareness. The reminder checklist yielded a significantly higher recording rate of the following: time that the injury had occurred, the consistency of the history, the compatibility of the injury with the history given, the consideration of the possibility of NAI, the general state and behaviour of the child, and the presence or absence of any other injuries. This study also showed that after introduction of the education and checklist the rate of referral for a further opinion regarding the possibility of NAI increased from $0 \%$ to $3 \%$.

Sidebotham and Pearce ${ }^{4}$ developed "child protection procedures" in emergency departments, which resulted in a list of the following five child risk indicators:

1. Three or more previous attendances

2. History inconsistent

3. History/examination inconsistent

4. Delay in presentation

5. Fracture or head injury in under 1 year old

Dr Sidebotham recommended that if number 5 was positive or a total of two positive indicators were recorded the case should be discussed with the paediatric registrar.

This study aims:
- To investigate if cases of possible NAI injury as identified using five risk indicators by Dr Sidebotham, give rise to any suspicions of child abuse

- To check if guidelines in relation to referral of cases of NAI are being complied with

\section{METHOD}

\section{Participants}

Participants were all children aged 16 years and under presenting with an injury at the emergency departments during the period July 2002-December 2002.

\section{Procedure}

A3 size posters were placed in both the staff and patient seating areas of Letterkenny General Hospital and Sligo General Hospital explaining that the public health department was investigating the prevalence of childhood accidents. These posters assured parents attending the emergency department with an injured child that the nurse and doctor completing questionnaires concerning their child was standard practice during the six month research period and enforced the fact that all data collected were anonymous. When a child under the age of 16 attended the emergency department with an injury or after swallowing a harmful substance, a questionnaire was attached to the medical recording card and the triage nurse completed section 1 and the attending doctor completed section 2. This study was based on investigations concerned with the identification of NAI carried out in the UK by Dr Sidebotham. To this end the five risk indicators identified by $\mathrm{Dr}$ Sidebotham were incorporated in the questionnaire. In addition, section 1 of the questionnaire obtained demographic information of age, sex, place of residence, time of attendance, who attended the emergency department with the injured child, what type of injury was presented, for example, cut, fracture, how the injury occurred, for example, sport, fall. The nurse also provided a written description of how the injury had occurred. The nurse was asked about subjective concerns and whom these concerns had been discussed with. In both section 1 and section 2, accident prevention information was obtained. The attending doctor provided information, in 
Table 1 Frequency of each indicator being positive for children presenting at the emergency departments

\begin{tabular}{ll}
\hline Risk indicator & $\begin{array}{l}\text { Number of cases } \\
\text { identified }\end{array}$ \\
\hline Inconsistency in history & 22 \\
Inappropriate delay & 42 \\
Fracture/head injury in child $<1$ year & 6 \\
Examination match the history & 4 \\
Greater than three attendances & 149
\end{tabular}

section 2, on the time the patient was seen, also information of the doctor's subjective concerns and with whom these concerns were discussed was sought. These results will be presented elsewhere. Section 1 of the questionnaire asked for information, from the triage nurse, on risk indicator numbers $1,2,4$, and 5 . The attending doctor completed information on the third risk indicator.

\section{RESULTS}

\section{Sample}

During the six month period 943 questionnaires were completed for children attending the emergency departments at both Sligo General Hospital and Letterkenny General Hospital. Both sections of the questionnaire were completed in 560 cases, 113 questionnaires had only the doctor's section completed and the section to be completed by the triage nurse was left blank. It is necessary for the nurse to complete the form to obtain information on the child and type of injury; consequently these cases were not included in the database. However, it must be noted that in none of these 113 cases were issues of child abuse raised. The section of the questionnaire to be completed by the doctor was left blank in 270 cases in which the nurse's section was completed. Because of the substantial information contained in the triage nurse section of the questionnaire these questionnaires were included in the final analysis. Thus the final dataset comprised of 830 cases.

\section{Demographics of the sample}

Sex was not recorded in 78 cases. Of the 752 cases in which sex was recorded 432 (57\%) were male and 320 (43\%) were female.

Five risk indicators of NAI were incorporated in the questionnaire; table 1 shows the frequency of cases with a reported positive indicator.

Table 1 indicates a large proportion (39\%) of children attending the emergency department with an injury have had three or more previous attendances. The present computer system in both hospitals fails to facilitate identification of date and clinical coding of previous attendances. Thus children attending more than three times could have attended numerous times with, for example, an asthma complaint and not necessarily an injury. The failure of the present computer system to provide a date of previous attendances could mean a child aged 14 attended three times while under the age of 6 and has not attended in the past eight years yet three attendances would be flagged up as a negative indicator when this is clearly not the case.

Dr Sidebotham recommends that a child with two or more positive risk indicators is a case that should warrant further concern. Table 2 illustrates the frequency of each combination of two positive risk indicators.

Table 2 indicates that one child under the age of 1 year had a possible fracture and also had an inconsistency in history. Another child under the age of 1 year also has a suspected fracture in conjunction with an inappropriate delay. Children under the age of 1 year are usually closely supervised at all times and as they are comparatively immobile until the age of 10-12 months, the incidence of "accidental" injuries should be low. Subsequently these children should have raised concerns in hospital staff because of the nature of the injury alone regardless of the additional positive risk indicator. However, this investigation revealed plausible explanations for the six children under 1 year, attending with a possible fracture or head injury.

This investigation also assessed the subjective concerns of the doctor and the nurse and it was shown that neither the doctor nor the nurse had subjective concerns about any of the patients who would have been identified by the positive risk indicators. However, both the doctor and nurse had concerns in three cases in addition the nurse alone had concerns in three other cases and the doctor alone had concerns in three additional cases. Thus, in total nine cases raised subjective concerns in either the nurse or doctor, or both. The hospitals have a protocol in place when dealing with suspected NAI and each member of staff has an identified superior with which the issue is to be discussed. All bar one of the cases, in which subjective concerns were raised, were discussed with a superior. The case, identified by the doctor as raising concerns, in which the doctor did not discuss the matter was that of a 9 year old boy accompanied by his parent who reported an "alleged assault by two other children and sustained an injury to his elbow from a spade handle". The doctor reported that the boy was beaten by twin brothers (friends), and gave advice for the boy to stop fighting and run away. This is clearly not a case of child abuse and subsequently there was no need to discuss the case with the designated superior. Thus in all cases in which subjective concern was raised the staff discussed it with the relevant work associate.

\section{Conclusions and recommendations}

This investigation highlights difficulties in the patient admission systems used in the emergency departments of the North Western Health Board. The present computerised system makes the assessment of dates and reasons for previous attendances a cumbersome task, which is impossible at the time of admission. This research suggests a need for a more informative computerised system, which flags up

Table 2 Frequency of each combination of two positive risk indicators for children presenting at the emergency departments

\begin{tabular}{|c|c|c|c|}
\hline Combination of risk indicators & Number & Combination of risk indicators & Number \\
\hline $\begin{array}{l}\text { Inconsistency and a } \\
\text { fracture in a child }<1\end{array}$ & 1 & $\begin{array}{l}\text { Inconsistency and greater than } \\
\text { three attendances }\end{array}$ & 2 \\
\hline $\begin{array}{l}\text { Inappropriate delay and a } \\
\text { fracture in child }<1\end{array}$ & 1 & $\begin{array}{l}\text { Inappropriate delay and greater } \\
\text { than three attendances }\end{array}$ & 8 \\
\hline $\begin{array}{l}\text { Examination match the history } \\
\text { and }>\text { than three attendances }\end{array}$ & 1 & & \\
\hline
\end{tabular}


the date and clinical code of the previous 10 attendances. This would facilitate the identification of children who have recently attended the emergency department with an injury, subsequently making the greater than three attendances a more reliable indicator. Taking this into consideration the use of Dr Sidebotham's indicators highlighted two cases with two or more positive indicators, however these cases failed to raise subjective concerns in the emergency department staff. Thus, suggesting that the recording of these risk indicators alone may be insufficient in investigating the possibility of identifying NAI in a busy emergency department.

It was shown that when subjective concerns were raised the staff did follow the protocol set out within each emergency department. However, the difficulties associated with the identification of child maltreatment and the substantial flow of patients through emergency departments dictates the need for a clear protocol of interagency communication, which is easily accessible and installed in the admissions department of the emergency department. The United Kingdom has acknowledged the necessity for emergency departments to have clear protocols for recognising and handling suspected abuse, subsequently local measures exist in UK hospitals, for example, the Royal United Hospital in Bath assesses children by way of checking the child protection register and pinpointing five main indicators of risk for possible abuse.

This investigation suggests that in conjunction with staff education and recording of the five indices of abuse there is a need for a computerised admission system and a computerised child protection register, which would be accessible by the appropriate emergency department staff when risk indicators are identified or when subjective concerns are raised.

\section{Authors' affiliations}

A McKinney, G Lane, F Hickey, North Western Health Board, Public Health Department, Letterkenny, Republic of Ireland

\section{REFERENCES}

1 Mace SE, Gerardi MJ, Dietrich AM, et al. Injury prevention and control in children. Ann Emerg Med 2001;38:405-14.

2 Kempe CH, Silverman FN, Steel BF, et al. The battered child syndrome. JAMA 1962;181:17-24.

3 Benger JR, McCabe SE. burns and scalds in pre-school children attending accident and emergency: accident or abuse? Emerg Med J 2001;18:172-4.

4 Sidebotham PD, Pearce AV. Audit of child protection procedures in accident and emergency department to identify children at risk of abuse. $B M J$ 1997;315:855-6. 